CARDIOLOGY

\title{
Prognostic Significance of Circulating Levels of Hepatocyte Growth Factor in Patients with Chagas' Disease and Idiopathic Dilated Cardiomyopathy
}

\author{
Yong Wang ${ }^{a}$ Maria da Consolação V. Moreira ${ }^{f}$ Adnan Khan $^{a}$ \\ Silvia Heringer-Walther ${ }^{g}$ Heinz-Peter Schultheiss ${ }^{b}$ Niels Wessel ${ }^{c}$ \\ Wolf-Eberhard Siems ${ }^{d}$ Thomas Walther ${ }^{\text {a, e }}$ \\ ${ }^{a}$ Department of Experimental Cardiology, Excellence Cluster Cardio-Pulmonary System, Justus-Liebig University,

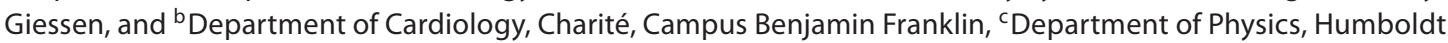

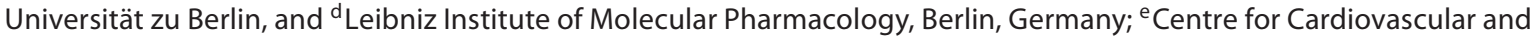 \\ Metabolic Research, Hull York Medical School, University of Hull, Hull, UK; ${ }^{f}$ Department of Internal Medicine, Federal \\ University of Minas Gerais, Belo Horizonte, and ${ }^{9}$ Instituto Metropolitano de Ensino Superior/Univaço, Ipatinga, Brazil
}

\section{Key Words}

Chagas' disease $\cdot$ Dilated cardiomyopathy $\cdot$ Heart failure $\cdot$

Hepatocyte growth factor

\begin{abstract}
Objectives: Hepatocyte growth factor (HGF) plays an important role in the improvement in cardiac function and remodeling in a variety of cardiovascular diseases. It is also a strong predictor of mortality in some heart failure (HF) patients. However, its prognostic value in patients with Chagas' disease (CD) or idiopathic dilated cardiomyopathy (DCM) remains to be investigated. Methods and Results: In this prospective cohort study, HGF concentrations were measured in patients with $C D(n=91), D C M(n=47)$, and control subjects $(n=25)$. While no difference was detected for patients with New York Heart Association class I-II, HGF was significantly increased in advanced HF patients (New York Heart Association class III-IV) in both CD and DCM groups, compared with healthy subjects. There was a strong correlation between HGF and left ventricular ejection fraction in CD patients. However, there was no correlation in patients with
\end{abstract}

\section{KARGER}

Fax +4161306 1234

E-Mail karger@karger.ch

www.karger.com
(C) 2012 S. Karger AG, Basel

$0008-6312 / 12 / 1214-0240 \$ 38.00 / 0$

Accessible online at:

www.karger.com/crd
DCM. Despite its strong correlation with left ventricular ejection fraction in CD patients, HGF failed to predict mortality and necessity for heart transplant in both $C D$ and DCM patients. Conclusions: Although HGF can be significantly increased in advanced HF patients with CD and DCM, its prognostic value for endpoints is minor. Therefore, the formerly described predictive power for HGF in HF might be restricted to specific etiologies of HF.

Copyright $\odot 2012$ S. Karger AG, Basel

\section{Introduction}

Regarded by the World Health Organization as one of the 13 most neglected tropical diseases in the world, Chagas' disease (CD) continues to be a serious economic, social and health problem in many Latin American countries [1]. CD is endemic throughout Central and South America where 16-18 million individuals are estimated to be infected and another 100 million are at risk of infection [2]. Furthermore, the recent influx of immigrants from countries endemic for the disease has meant that 
CD is fast becoming an important health issue in the USA and Canada, in many parts of Europe like Spain and France, and the Western Pacific, where an increasing number of infected individuals have been identified [3].

$\mathrm{CD}$ has two successive phases: acute and chronic. The acute phase lasts 6-8 weeks. Once the acute phase subsides, most infected patients recover an apparently healthy state, in which no organ damage can be detected using the current standard methods of clinical diagnosis. The infection can only be confirmed by serological or parasitological tests. This form of the chronic phase of CD is known as the intermediate form [4]. Most patients remain in this part of the chronic stage indefinitely.

However, several years after entering the chronic phase, $25-30 \%$ of infected individuals, depending on the geographical area, will develop irreversible lesions of the autonomic nervous system in the heart, esophagus and colon, and of the peripheral nervous system. The chronic phase lasts for the duration of the infected individual's life [5].

Cardiac involvement, the most frequent and serious manifestation of chronic $\mathrm{CD}$, typically produces atrial and ventricular arrhythmias, conduction defects, heart failure (HF), cardiomegaly, apical aneurysms, thromboembolic complications, or sudden death. CD remains the leading cause of heart disease in Latin America. Once HF develops, life expectancy is reduced to a few years. The mortality rate caused by sudden death is about $40 \%$ in CD patients with HF. Thus, sudden death and HF together account for most of the deaths that occur in patients with CD.

$\mathrm{HF}$ due to $\mathrm{CD}$ and dilated cardiomyopathy from other causes (DCM) is often characterized by a progressive remodeling of the left ventricular (LV) chamber. Ventricular remodeling is related to various processes such as cardiomyocyte loss due to necrosis or to apoptosis, hypertrophic responses of cardiomyocytes in the surviving regions of the left ventricle, angiogenesis, and an architectural rearrangement of the extracellular matrix $[6,7]$.

Hepatocyte growth factor (HGF), originally identified as a potent mitogen for hepatocytes, is reported to have mitogenic, antiapoptotic, angiogenic, and antifibrotic activities in various cell types [8]. Recent experimental studies have implicated HGF in the process of ventricular remodeling and have suggested its cardioprotective effects [9-12]. The endogenous HGF system protects the heart via an antiapoptotic effect on cardiomyocytes and attenuates the development of HF with increased angiogenesis and decreased apoptosis and fibrosis [7].

There has been no previous study regarding plasma HGF measurements in CD patients and in a well-defined group of patients with DCM. Thus, the aim of our study was: (1) to measure plasma HGF concentration in CD patients and compare it with those in healthy human volunteers, evaluating the measurement of HGF as a possible screening tool for ventricular dysfunction; (2) to investigate a possible correlation with the functional New York Heart Association (NYHA) class and LV ejection fraction (LVEF); (3) to evaluate the prognostic impact of plasma HGF for cardiac death and the necessity for heart transplant in CD; and (4) to compare the circulating HGF levels of CD patients with those having DCM due to other causes, characterizing $\mathrm{HGF}$ as a possible diagnostic marker to differentiate between the two types of HF.

\section{Methods}

\section{Patients}

An institutional review committee approved the study, and all patients gave written consent. The study population consisted of a prospective cohort of 138 patients from the Heart Failure Centre of Felicio Rocho Hospital, Brazil, enrolled between July 2001 and January 2005. Ninety-one consecutively recruited patients with at least 2 positive serologies for CD (the CD group) and 47 patients with negative serology for CD (the idiopathic DCM group) were studied and compared with 25 adjusted gender- and age-matched healthy subjects.

Clinical data including medical history, physical examination, resting electrocardiogram (ECG), and echocardiographic parameters were collected by the same investigator, as reported previously $[5,13,14]$. Systolic LV dysfunction was defined by an LVEF of $<50 \%$, as assessed by transthoracic echocardiography (Teichholz method).

Other structural cardiac diseases and comorbidities were excluded in both groups by medical history, physical examination, ECG, M-mode, 2-dimensional and Doppler echocardiography. Patients with valvular heart disease, coronary artery disease, congenital disease, acute myocarditis, hypertensive disease, renal failure (plasma creatinine $>0.2 \mathrm{mmol} / \mathrm{l}$ ), chronic pulmonary disease, hepatic cirrhosis, active infection, and endocrine disease have not been included into the study. Patients with ventricular dysfunction received standard pharmacologic therapy according to the NYHA functional class and were clinically stable for at least 30 days.

Patients with advanced refractory HF were considered to be heart transplant candidates and were listed for the procedure in the absence of a contraindication. The control group consisted of healthy subjects attending the hospital clinics for a physical checkup. They had no cardiac symptoms or history, no comorbidities, and were not taking any medication.

The study patients were prospectively defined and subdivided into 5 groups: group $1(\mathrm{n}=46), \mathrm{CD}$ without systolic ventricular dysfunction (LVEF $>50 \%)$; group $2(\mathrm{n}=22)$, CD with ventricular systolic dysfunction (LVEF < 50\%) in NYHA classes I-II; group 3 $(\mathrm{n}=23), \mathrm{CD}$ with ventricular systolic dysfunction (LVEF <50\%) in NYHA classes III-IV; group $4(\mathrm{n}=21)$, DCM with ventricular systolic dysfunction (LVEF $<50 \%$ ) in NYHA classes I-II; and group $5(\mathrm{n}=26)$, DCM with ventricular systolic dysfunction (LVEF < 50\%) in NYHA classes III-IV. 
Patients were followed for incidences of cardiac death or heart transplant from the time the blood sample was obtained for HGF analysis until endpoints were reached or until the follow-up closing date in January 2006. Long-term follow-up of each patient was conducted by medical visit or telephone interview every 3 months. For those hospitalized during follow-up, the hospital records were reviewed. For all subjects who died, the nearest relative was contacted.

\section{Plasma Samples and HGF Measurement}

To measure HGF in plasma, blood samples $(10 \mathrm{ml})$ were taken from an antecubital vein and transferred into tubes containing ethylenediaminetetraacetic acid. Immediately after sampling, plasma was separated by centrifugation at $4,000 \mathrm{~g}$ for $10 \mathrm{~min}$, frozen at $-80^{\circ} \mathrm{C}$ for further analysis. Before collecting blood samples, patients had been treated for at least 3 months, and their clinical symptoms were stable.

Plasma concentrations of HGF were measured using the BioPlex system that combined the principle of a sandwich immunoassay with the Luminex fluorescent-bead-based technology (BioRad Laboratories) [15].

\section{Statistical Analysis}

All data were expressed as the mean \pm standard error of the mean (SEM). We tested associations between plasma HGF concentrations and clinical variables using the Mann-Whitney $U$ test. Because data were normally distributed, Pearson's correlation coefficient ( $r$ ) was used to analyze the correlation between the 'investigated' HGF in CD and DCM patient groups - they were tested against the null hypothesis that the correlation coefficient is 0 . A p value $<0.05$ was defined as significant. We used the Kaplan-Meier analysis to compare the survival or necessity for heart transplantation of patients with CD and DCM depending on different HGF concentrations.

\section{Results}

Baseline characteristics of patients and controls according to the NYHA functional class, ECG, echocardiographic parameters, and medications are summarized in table 1. The mean duration of follow-up was 36.9 months (range 13-54). By the end of the study, the survival status of all patients was known; 29 patients had died and 12 patients received a heart transplant.

Systolic blood pressure was significantly altered in patients in the CD group and in patients in the DCM group with advancing HF in comparison with the control group or patients with CD without systolic ventricular dysfunction. Table 1 also illustrates increased impairment of cardiac function in both patients with CD and in patients with DCM with increasing NYHA class.

All patients with ventricular dysfunction received standard medical therapy according to their functional classes and the treatment guideline recommendations, as summarized in table 1 . Almost all patients with ventricular dysfunction were given angiotensin-converting enzyme inhibitors (ACEIs) or angiotensin receptor blockers; 21 patients with DCM were taking carvedilol, but only 3 patients with CD were receiving $\beta$-blocker therapy. This lack in $\beta$-blocker treatment in patients with CD resulted from missing experience with $\beta$-blockers in $C D$, because all the major trials on HF exclude patients with $\mathrm{CD}$. Moreover, this disease is very often characterized by bradyarrhythmias and advanced atrioventricular block, which makes it difficult to use $\beta$-blockers.

The individuals in the control group presented with normal and homogenous HGF levels (240.2 $\pm 27.36 \mathrm{pg} /$ $\mathrm{ml}$ ), as described in the literature (fig. 1a) [16,17]. Patients with $\mathrm{CD}$ without systolic ventricular dysfunction have been characterized by unaltered HGF levels (242.5 \pm $16.88 \mathrm{pg} / \mathrm{ml})$. While patients with DCM and CD in NYHA classes I and II were also characterized by unaltered HGF plasma levels, these plasma concentrations significantly increased in patients with NYHA classes III and IV ( $p<0.001$; fig. 1a).

Because we were interested in finding out whether the HGF levels are related to impaired cardiac function in patients with CD and patients with DCM, we first performed a correlation analysis to detect a possible correlation between HGF and LVEF. While both parameters correlated with high significance in CD patients of NYHA I-IV ( $p<0.01$ ) (fig. 1b), there was no correlation in the patients with DCM ( $p=0.1498$ ) (fig. 1c). Moreover, the data summarized in figure $1 \mathrm{~d}$ show that half of the monitored cardiac parameters recorded by echocardiography (table 1) evidenced a correlation with the circulating HGF concentration in patients with $\mathrm{CD}$, with the correlation reaching significance for the parameters: LVEF ( $\mathrm{p}=$ $0.0013)$, LV systolic dysfunction ( $\mathrm{p}=0.017)$, and LV endsystolic volume $(\mathrm{p}=0.024)$. However, there was no significant correlation between HGF and any echocardiographic parameters in patients with DCM.

To find out what led to this discrepancy in correlation between HGF and echocardiographic parameters, we compared the medical treatment for patients with $C D$ and DCM. Only 3 patients from the CD group received $\beta$-blockers, but approximately half of DCM patients took them. To exclude the possible role of $\beta$-blockers on HGF in DCM patients preventing a significant correlation as seen in patients with $C D$, we further analyzed the subpopulations without $\beta$-blocker treatment. As for the whole set (fig. 1a), in both CD and DCM patients who were not on $\beta$-blocker therapy, there was still no significant alteration in plasma HGF levels in NYHA classes I 
Table 1. Patient characteristics

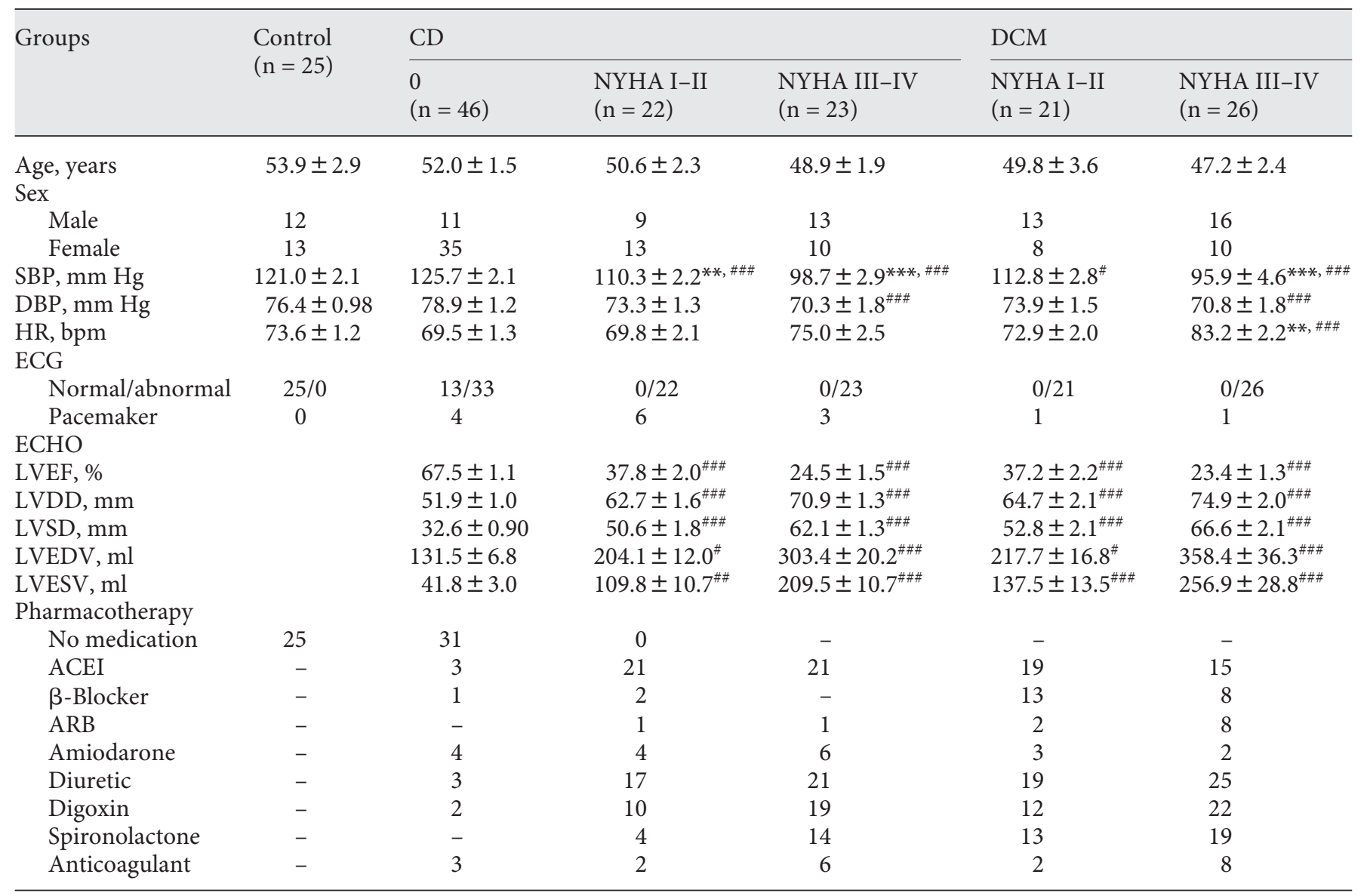

Data are given as the average \pm SEM. ${ }^{* *} \mathrm{p}<0.01,{ }^{* * *} \mathrm{p}<0.001$ vs. control; ${ }^{\#} \mathrm{p}<0.05,{ }^{\# \#} \mathrm{p}<0.01,{ }^{\# \# \#} \mathrm{p}<0.001$ vs. the CD 0 . Abnormal ECG includes arrhythmias, flutter and atrial fibrillation, any bundle branch block, atrioventricular block and atrial/ventricular hypertrophy. SBP = Systolic blood pressure; DBP = diastolic blood pressure; HR = heart rate; $\mathrm{ECHO}=$ echocardiography; LVDD = LV diastolic diameter; LVSD = LV systolic diameter; LVEDV = LV end-diastolic volume; LVESV = LV end-systolic volume; ARB = angiotensin AT1 receptor blocker.

and II, but the plasma concentrations increased significantly in patients with NYHA classes III and IV as compared to controls ( $p<0.001$; fig. 2a). Notably, the DCM patients with NYHA classes I and II had a trend for elevation in HGF levels. Furthermore, LVEF still evidenced a significant correlation with the circulating HGF concentration in $\mathrm{CD}$ patients without $\beta$-blocker therapy $(\mathrm{p}<$ 0.01 ; fig. $2 \mathrm{~b}$ ), while in DCM patients without $\beta$-blocker therapy, the correlation was even impaired $(\mathrm{p}=0.5058)$ compared to the whole DCM group ( $\mathrm{p}=0.1498)$ (fig. 2c).

There were deaths from pump failure (NYHA class I-II, $\mathrm{n}=6$; NYHA class III-IV, $\mathrm{n}=11$ ) and heart transplants (NYHA class IV, $n=6$ ) in the CD group, whereas in the DCM group, 6 patients underwent a heart trans- plantation (NYHA class II, $\mathrm{n}=1$; NYHA class III, $\mathrm{n}=1$; NYHA class IV, $\mathrm{n}=4$ ) and 12 patients died (NYHA class $\mathrm{I}, \mathrm{n}=1$; NYHA class III, $\mathrm{n}=11$ ). The receiver operating characteristic curve was generated to find a cut-off value for plasma HGF in CD patients (fig. 3a). To find out whether the HGF cut-off value $(314.3 \mathrm{pg} / \mathrm{ml})$ has any prognostic potency for the lethality of patients with CD and DCM, Kaplan-Meier curves were generated using this cut-off value to divide the patients with $\mathrm{CD}$ and patients with DCM into 2 subgroups. Classical statistics visualized no significant predictive value of HGF plasma concentration for risk in lethality or the necessity for heart transplantation in both CD patients (NYHA I-IV) and DCM patients (fig. 3b, c). 


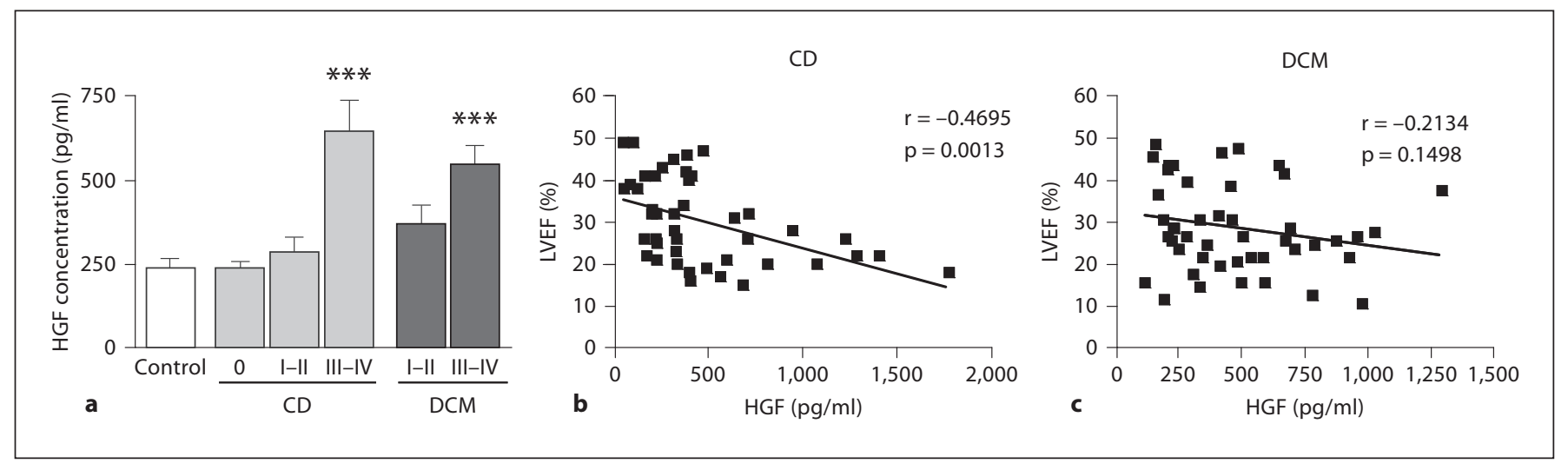

Fig. 1. Circulating HGF concentrations in patients with $\mathrm{CD}$ and idiopathic DCM as well as in control subjects. a HGF was measured in control healthy subjects and in patients with CD and DCM, which were further divided into three or two groups according to NYHA classes. ${ }^{* * *} \mathrm{p}<0.001$ compared to healthy subjects. Correlation between HGF concentrations and LVEF in CD (b) and DCM (c) patients was analyzed by Pearson correlation. Correlation between HGF and other echocardiographic parameters was summarized in patients with CD and DCM from NYHA I-IV (d). LVDD = LV diastolic diameter; LVSD = LV systolic diameter; LVEDV = LV end-diastolic volume; LVESV = LV end-systolic volume.

\begin{tabular}{|c|c|c|c|c|}
\hline & \multicolumn{2}{|c|}{ CD (NYHA I-IV) } & \multicolumn{2}{|c|}{ DCM (NYHA I-IV) } \\
\hline & $r$ & $\mathrm{p}$ & $r$ & $\mathrm{p}$ \\
\hline LVDD & 0.222 & 0.139 & 0.137 & 0.365 \\
\hline LVSD & 0.351 & 0.017 & 0.191 & 0.204 \\
\hline LVEDV & 0.136 & 0.398 & 0.221 & 0.166 \\
\hline LVESV & 0.352 & 0.024 & 0.285 & 0.071 \\
\hline & & & & \\
\hline
\end{tabular}

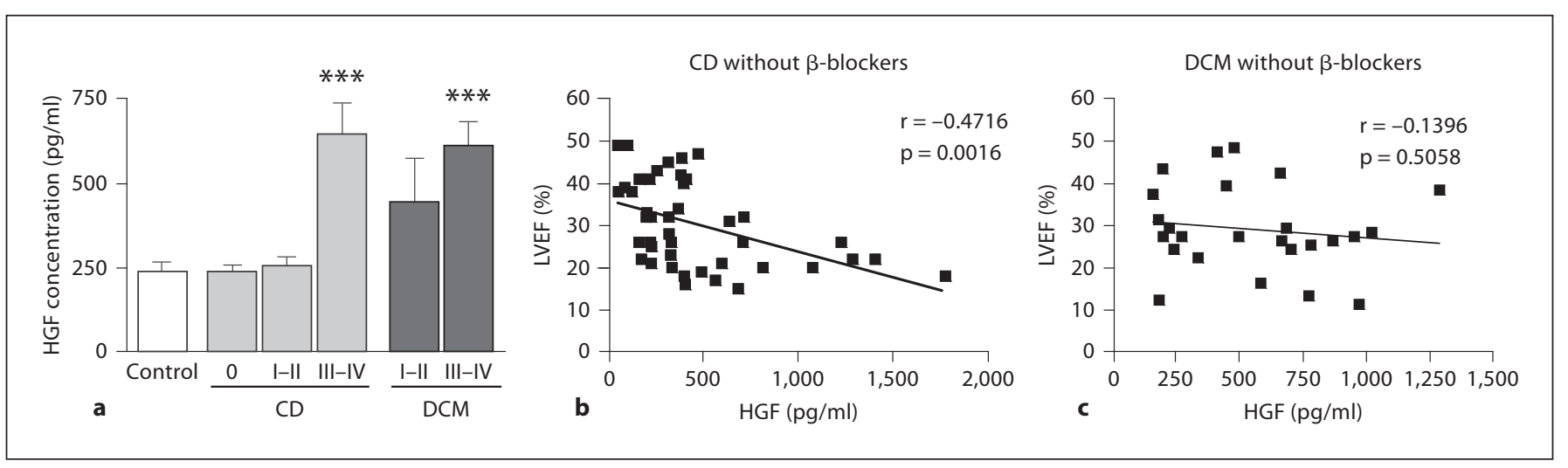

Fig. 2. HGF in patients without $\beta$-blocker treatment. a Concentrations of HGF in healthy subjects and CD and DCM patients without taking $\beta$-blockers. Correlations between HGF concentration and LVEF in CD patients (b) and DCM patients (c) without $\beta$-blocker treatment were analyzed by Pearson analysis. ${ }^{* *} \mathrm{p}<0.001$ compared to healthy subjects.

\section{Discussion}

Despite recent advances in diagnosis and management of HF, it is still an important public health problem worldwide. Therefore, it is very important to identify HF patients with a high risk to develop cardiovascular events in time and to prevent their onsets by prompt and proper treatments. Plenty of clinical and molecular markers have been identified to have prognostic potency, and a combination of several markers has been confirmed to have additive beneficial effects for risk prediction [18]. However, it is still desirable to discover novel biomarkers for risk stratification in particular HF populations with different etiology.

HGF is such a new molecular marker to predict mortality in congestive HF patients [7, 19, 20]. It was even discussed that HGF may become the next B-type natriuretic 


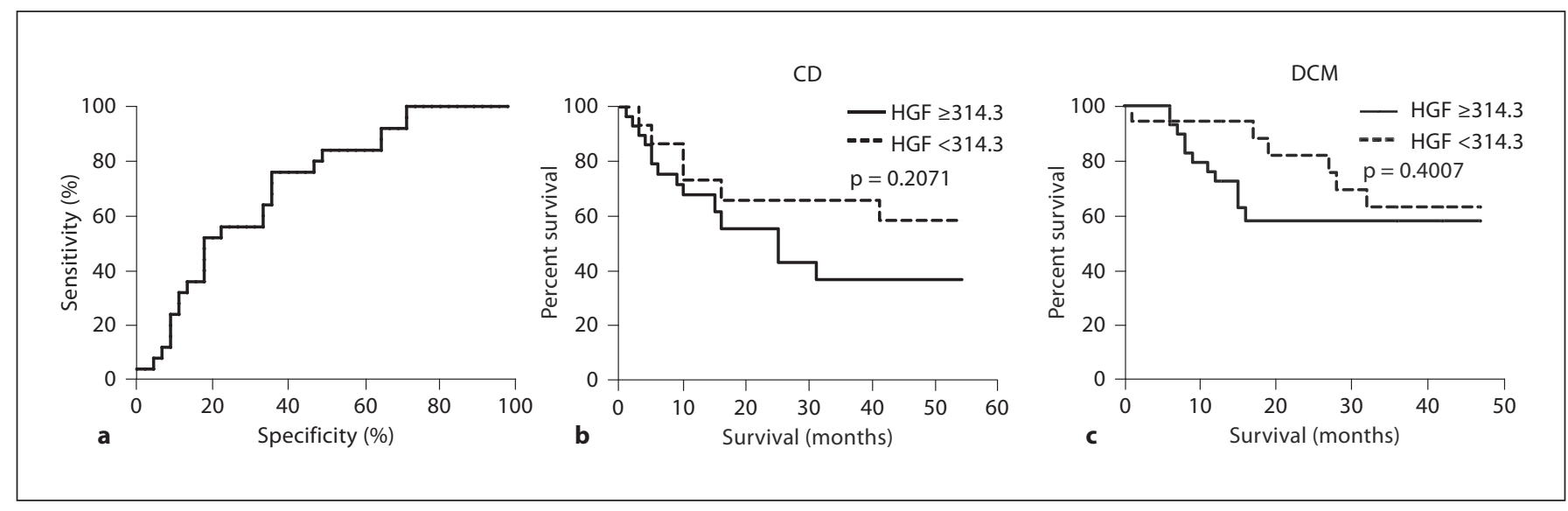

Fig. 3. Prognostic value of HGF in CD and DCM patients. The receiver operating characteristic curve was used to define best HGF cut-off value with best sensitivity and specificity based on CD patients with NYHA classes I-IV (a). Kaplan-Meier survival curves were constructed to compare the survival rate in patients with CD (b) and DCM (c), respectively. Statistical analysis was carried out by logrank test.

peptide which is the most commonly used biomarker for cardiovascular diseases [21]. In the present study, we focused on two specific HF patient populations with HF caused by either CD or idiopathic DCM, which were not included in previous clinical trials. Plasma HGF was identified to be significantly increased in patients with advanced HF (NYHA class III-IV) but not in patients with mild to moderate severity (NYHA class I-II). However, in our present study, HGF concentration in HF patient plasma was lower than the values reported by Lamblin et al. [7] and Rychli et al. [19]. One explanation could be the different methods used. In our study, we used the Bio-Plex Pro $^{\mathrm{TM}}$ Assays system (Bio-Rad), while in the other two studies, the traditional ELISA kit for HGF was used. However, the most possible reason is the different composition of recruited patients and treatments they received. It was confirmed by different clinical trials that circulating HGF concentration was positively correlated with age and clinical severity $[7,19]$. In the report by Rychli et al. [19], the median age of recruited patients is even 75 years and only advanced HF patients were included. However, the average age of HF patients in our present study is only 49 years and patients from NYHA class I-IV were included.

It was reported that HGF concentration was markedly increased in patients with congestive $\mathrm{HF}$ at admission and gradually returned to normal level during hospitalization [20]. Therefore, it is reasonable to assume that different treatment may also influence HGF concentration. Since in the study described by Rychli et al. [19] only hospitalized HF patients with clinical signs and symptoms of car- diac decompensation (NYHA class III-IV) were included, and blood was collected in the morning of the day of discharge before intake of medication, this might explain the higher concentration and positive predictive value. In another study, peripheral blood was collected for HGF measurement at the time of entry into the study [7]. In our present study, the heart function of all patients was compensated and they were treated with maximal tolerated doses of ACEIs, angiotensin receptor blockers, and others, for at least 3 months before the blood was obtained. Since the dose of ACEIs had been discovered to be inversely correlated with HGF concentration [19], and in the present study, most of the patients received ACEI therapy, this may also be part of the reasons why the HGF concentration measured was lower than previously described.

Although CD lead to typical DCM, it is not clear why there is a close correlation between HGF and echocardiographic parameters in CD patients but not in idiopathic DCM patients. We found that $\beta$-blocker treatment was not responsible for this discrepancy. It would be very interesting if a different DCM etiology may have an impact on the generation or the metabolism of HGF, and thus for the concentration of circulating HGF. If this can be proven, it might change our general understanding of dilated cardiomyopathy, since it is generally believed that the phenotype of dilated cardiomyopathy itself but not the causes for the phenotype determine the regulation of biomarkers of interest. However, further investigation with more patients per specific etiology is required to clarify such mechanisms. 
Although HGF had been identified to be a predictor of mortality in HF patients $[7,19]$, it cannot be used to predict primary endpoints in CD and DCM patients in our present study. However, our data are not per se in contradiction to other reports. In one study, subgroup analysis revealed that HGF was a strong predictor only in ischemic HF patients but not in non-ischemic patients [19]. In another study, over the time of follow-up, the percentage of ischemic etiology was significantly higher in patients with events compared to event-free patients [7]. However, in our present study, patients with coronary artery diseases were excluded. It seems that HGF concentration is mainly regulated in cardiac ischemic condition. This idea is further supported by the finding of Zhu et al. [22] that HGF was released after acute myocardial infarction.

In summary, although HGF concentration was significantly increased in advanced HF patients with $\mathrm{CD}$ and idiopathic DCM, our preliminary results indicate that it cannot be used to predict mortality or heart transplant in these patients. In combination with other reports, the conclusion can be made that etiology has to be taken into consideration when discussing the promising prognostic potency of HGF values in HF patients.

\section{Acknowledgement}

This study was supported by a DAAD and CAPES grant (415-br-probral/po-D/08/11632). S.H.-W. was supported by a fellowship of the Humboldt foundation.

\section{Conflict of Interest}

The authors declare that there are no financial interest conflicts related to this paper.

\section{References}

$>1$ Hotez PJ, Molyneux DH, Fenwick A, Kumaresan J, Sachs SE, Sachs JD, Savioli L: Control of neglected tropical diseases. N Engl J Med 2007;357:1018-1027.

$>2$ Moncayo A, Silveira AC: Current epidemiological trends for Chagas disease in Latin America and future challenges in epidemiology, surveillance and health policy. Mem Inst Oswaldo Cruz 2009;104(suppl 1):17-30.

$\checkmark 3$ Parker ER, Sethi A: Chagas disease: coming to a place near you. Dermatol Clin 2011;29: 53-62.

$\checkmark 4$ Bern C: Antitrypanosomal therapy for chronic Chagas' disease. N Engl J Med 2011; 364:2527-2534.

$>5$ Wang Y, Moreira Mda C, Heringer-Walther S, Ebermann L, Schultheiss HP, Wessel N, Siems WE, Walther T: Plasma ACE2 activity is an independent prognostic marker in Chagas' disease and equally potent as BNP. J Card Fail 2010;16:157-163.

$\checkmark 6$ Cohn JN, Ferrari R, Sharpe N: Cardiac remodeling - concepts and clinical implications: a consensus paper from an international forum on cardiac remodeling. Behalf of an International Forum on Cardiac Remodeling. J Am Coll Cardiol 2000;35:569-582. 7 Lamblin N, Susen S, Dagorn J, Mouquet F,
Jude B, Van Belle E, Bauters C, de Groote P: Prognostic significance of circulating levels of angiogenic cytokines in patients with congestive heart failure. Am Heart J 2005;150: 137-143.

$>8$ Matsumoto K, Nakamura T: Emerging multipotent aspects of hepatocyte growth factor. J Biochem 1996;119:591-600.

>9 Yang ZJ, Xu SL, Chen B, Zhang SL, Zhang YL, Wei W, Ma DC, Wang LS, Zhu TB, Li CJ, Wang
H, Cao KJ, Gao W, Huang J, Ma WZ, Wu ZZ: Hepatocyte growth factor plays a critical role in the regulation of cytokine production and induction of endothelial progenitor cell mobilization: a pilot gene therapy study in patients with coronary heart disease. Clin Exp Pharmacol Physiol 2009;36:790-796.

10 Yang ZJ, Chen B, Sheng Z, Zhang DG, Jia EZ, Wang W, Ma DC, Zhu TB, Wang LS, Li CJ, Wang $\mathrm{H}$, Cao KJ, Ma WZ: Improvement of heart function in postinfarct heart failure swine models after hepatocyte growth factor gene transfer: comparison of low-, mediumand high-dose groups. Mol Biol Rep 2010;37: 2075-2081.

11 Rastogi S, Guerrero M, Wang M, Ilsar I, Sabbah MS, Gupta RC, Sabbah HN: Myocardial transfection with naked DNA plasmid encoding hepatocyte growth factor prevents the progression of heart failure in dogs. Am J Physiol Heart Circ Physiol 2011;300:H1501-H1509.

12 Jayasankar V, Woo YJ, Bish LT, Pirolli TJ, Chatterjee S, Berry MF, Burdick J, Gardner TJ, Sweeney HL: Gene transfer of hepatocyte growth factor attenuates postinfarction heart failure. Circulation 2003;108(suppl 1): II230-II236.

13 Moreira Mda C, Heringer-Walther S, Wessel N, Moreira Ventura T, Wang Y, Schultheiss HP, Walther T: Prognostic value of natriuretic peptides in Chagas' disease: a 3-year follow-up investigation. Cardiology 2008;110: 217-225.

14 Wang Y, Moreira MC, Heringer-Walther S, Khan A, Schultheiss HP, Wessel N, Siems WE, Walther T: Does the aminopeptidase A (APA) have prognostic and diagnostic value in Chagas' disease and other dilated cardio- myopathies? J Cardiovasc Pharmacol 2011; 58:374-379.

15 Fu Q, Zhu J, Van Eyk JE: Comparison of multiplex immunoassay platforms. Clin Chem 2010;56:314-318.

16 Gohji K, Nomi M, Niitani Y, Kitazawa S, Fujii A, Katsuoka Y, Nakajima M: Independent prognostic value of serum hepatocyte growth factor in bladder cancer. J Clin Oncol 2000;18:2963-2971.

17 Sato T, Yoshinouchi T, Sugimoto T, Sakamoto T, Fujieda H, Murao S, Sato H, Ohe T: Prognostic value of serum hepatocyte growth factor in patients with acute coronary syndromes. Jpn Circ J 1999;63:583-588.

18 Clerico A, Vittorini S, Passino C, Emdin M New and emerging biomarkers of heart failure. Crit Rev Clin Lab Sci 2009;46:107-128.

19 Rychli K, Richter B, Hohensinner PJ, Kariem Mahdy A, Neuhold S, Zorn G, Berger R, Mortl D, Huber K, Pacher R, Wojta J, Niessner A, Hulsmann M: Hepatocyte growth factor is a strong predictor of mortality in patients with advanced heart failure. Heart 2011;97:1158-1163.

20 Ueno S, Ikeda U, Hojo Y, Arakawa H, Nonaka M, Yamamoto K, Shimada K: Serum hepatocyte growth factor levels are increased in patients with congestive heart failure. J Card Fail 2001;7:329-334.

21 Katz JN, Drazner MH: Assessing prognosis in heart failure: is hepatocyte growth factor the next B-type natriuretic peptide? Am Heart J 2005;150:1-3.

22 Zhu Y, Hojo Y, Ikeda U, Shimada K: Production of hepatocyte growth factor during acute myocardial infarction. Heart 2000;83: $450-455$. 